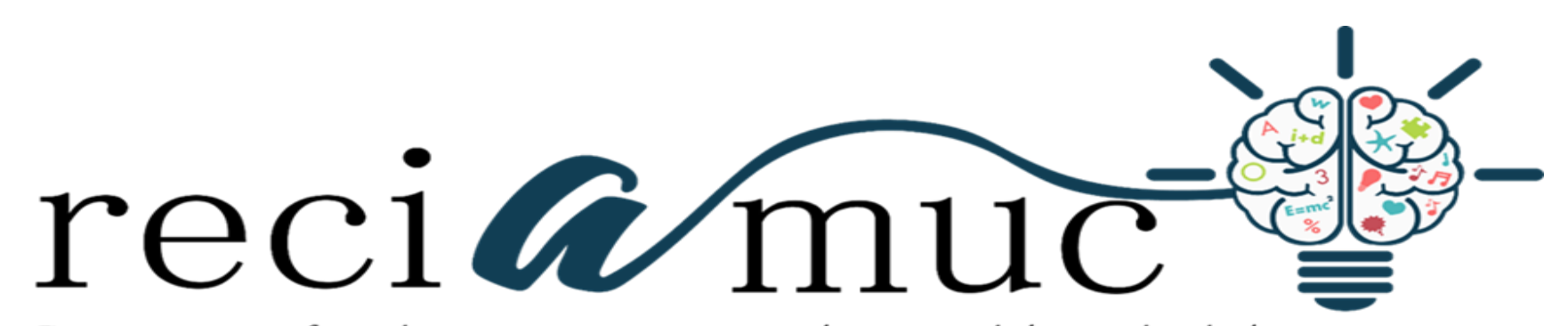

Revista científica de investigación actualización del mundo de las ciencias

Nancy Roció Castillo Castro a; Xavier Francisco Flores Torres ${ }^{\text {b; }}$ Víctor Hugo Navas Naranjo ${ }^{\mathrm{c}}$

Análisis de los principales instrumentos económicos implementados en la política ambiental del Ecuador

Analysis of the main economic instruments implemented in the environmental policy of Ecuador

Revista Científica de Investigación actualización del mundo de las Ciencias. Vol. 3 núm., 1, enero, ISSN: 2588-0748, 2018, pp. 635-652

DOI: 10.26820/reciamuc/3.(1).enero.2019.635-652

URL: http://reciamuc.com/index.php/RECIAMUC/article/view/251

Código UNESCO: 59 Ciencia Política

Tipo de Investigación: Artículo de Revisión

Editorial Saberes del Conocimiento

Recibido: 10/12/2018Ａceptado: 20/01/2019Ｐublicado: 30/01/2019

Correspondencia: nancy.castilloc@ug.edu.ec

a. Magister en Finanzas y Proyectos Corporativos; Economista; Universidad de Guayaquil; Guayaquil, Ecuador; nancy.castilloc@ug.edu.ec

b. Master Universitario en Desarrollo Económico y Políticas Publicas; Economista; Universidad de Guayaquil; Guayaquil, Ecuador; xavier.florest@ug.edu.ec

c. Economista; Universidad de Guayaquil; Guayaquil, Ecuador; victor.navasn@ug.edu.ec 


\section{Análisis de los principales instrumentos económicos implementados en la política ambiental del Ecuador}

Vol. 3, núm. 1., (2019)

Nancy Roció Castillo Castro; Xavier Francisco Flores Torres; Víctor Hugo Navas Naranjo

\section{RESUMEN}

El presente análisis describe la estructura, evolución y desempeño de los ingresos y gastos del sector público en materia ambiental, así como los principales instrumentos económicos implementados en la política ambiental del Ecuador, para sustentar las tareas de financiamiento de acciones ambientales y el cumplimiento de los objetivos de conservación de los recursos naturales.

El fin del presente estudio es analizar la fuente y el uso de los recursos destinados al desarrollo sostenible en el Ecuador durante los últimos años, tomando como aspecto principal la sostenibilidad del nivel del gasto y la inversión, así como en su utilización, además tomando como referencia los principales instrumentos económicos que sirven de base para establecer parte del financiamiento al desarrollo sostenible y el cumplimiento de los estándares de servicios asociados a la calidad ambiental y preservación de los recursos naturales.

Palabras Claves: Instrumentos Económicos; Política Ambiental; Desarrollo Sostenible. 


\title{
Análisis de los principales instrumentos económicos implementados en la política ambiental del Ecuador
}

Vol. 3, núm. 1., (2019)

Nancy Roció Castillo Castro; Xavier Francisco Flores Torres; Víctor Hugo Navas Naranjo

\begin{abstract}
The present analysis describes the structure, evolution and performance of public sector revenues and expenditures in environmental matters, as well as the main economic instruments implemented in Ecuador's environmental policy, to support the tasks of financing environmental actions and compliance with the conservation objectives of natural resources.

The purpose of this study is to analyze the source and use of the resources destined to sustainable development in Ecuador during the last years, taking as main aspect the sustainability of the level of the expense and the investment, as well as in its use, besides taking as reference the main economic instruments that serve as a basis to establish part of the financing for sustainable development and compliance with the standards of services associated with environmental quality and preservation of natural resources.
\end{abstract}

Key Words: Economic Instruments; Environmental Policy; Sustainable development. 


\section{Análisis de los principales instrumentos económicos implementados en la política ambiental del Ecuador}

Vol. 3, núm. 1., (2019)

Nancy Roció Castillo Castro; Xavier Francisco Flores Torres; Víctor Hugo Navas Naranjo

\section{Introducción.}

Pese a tener una superficie relativamente pequeña Ecuador es considerado como uno de los países más ricos en biodiversidad y ecosistemas de todo el mundo, su posición privilegiada y la presencia de la cordillera de los Andes, determinan la existencia de una gran variedad de bosques y microclimas.

Esta gran diversidad de climas, ha permitido al país disponer de una inmensa variedad de recursos que permiten abastecer las necesidades de consumo de los ecuatorianos y de muchos otros países del mundo.

El crecimiento económico del país puede ser medido por distintas variantes (crecimiento del PIB, productividad, nivel de educación de la población, las tasas de desempleo, etc.) para diagnosticar si en un determinado periodo de tiempo se ha incrementado el nivel de vida de la población.

Considerando que la mayor ventaja con la que cuenta el país es su biodiversidad, es fundamental saberla aprovechar de manera adecuada, mediante su conservación y su uso sustentable.

Hoy le corresponde esbozar la nueva política pública al gobierno de Lenìn Moreno, en primer lugar, se debe trabajar en productos que sean competitivos y señalar nuevas estrategias que se apunten a un mercado global. 


\section{Análisis de los principales instrumentos económicos implementados en la política ambiental del Ecuador}

Vol. 3, núm. 1., (2019)

Nancy Roció Castillo Castro; Xavier Francisco Flores Torres; Víctor Hugo Navas Naranjo

El gobierno debe apuntar, entonces a las políticas de sostenibilidad de la Asamblea General de las Naciones Unidas haciendo especial hincapié en explora el sector turístico y así fortalecer los tres pilares de la sostenibilidad (económico, social y medioambiental).

\section{Base teórica}

La incorporación de la sustentabilidad en los paradigmas de desarrollo es un asunto de esencial importancia para la supervivencia de la humanidad. Para ello es necesario conjugar el crecimiento y el desarrollo económico y social con una administración eficiente y racional de los recursos naturales que permita a la población tener acceso a niveles crecientes de bienestar y calidad de vida. La sustentabilidad ambiental exige, además, el aprovechamiento y transformación racional de los recursos naturales y el medio ambiente preservándolos para las generaciones futuras.

\section{El crecimiento económico y su medición}

El indicador utilizado comúnmente para expresar sintéticamente el desempeño de la economía es el producto interno bruto (PIB). Este agregado muestra el valor de la producción de bienes y servicios finales generados por la economía en un periodo dado. El destino del PIB es el consumo final de hogares y de gobierno, la inversión bruta y la balanza comercial con el exterior. El PIB refleja la generación del ingreso y la retribución al capital y al trabajo; es un indicador clave en el sistema de cuentas nacionales y es muy popular en la elaboración de modelos matemáticos y econométricos para pronosticar el desempeño de la economía. El PIB goza de gran popularidad. En el discurso político es manejado en las campañas electorales para divulgar metas que enuncian prosperidad y desarrollo confundiendo estas categorías. Los gobiernos ya en 


\section{Análisis de los principales instrumentos económicos implementados en la política ambiental del Ecuador}

Vol. 3, núm. 1., (2019)

Nancy Roció Castillo Castro; Xavier Francisco Flores Torres; Víctor Hugo Navas Naranjo

el poder, suelen exponer sus éxitos divulgando la evolución del PIB, siempre que el resultado les sea favorable.

Crecimiento, desarrollo económico y social

Entre los conceptos crecimiento económico, desarrollo económico y desarrollo social, pueden establecerse algunas diferencias esenciales, aunque es preciso plantear que existe una interrelación dialéctica que los condiciona. El desarrollo social, aunque no es una relación de estricta precedencia, requiere de cierto grado de desarrollo económico para alcanzarlo. Si la sociedad no cuenta con los excedentes que necesita la población para mantener su aumento natural sin lesionar el per capital con relación al producto creado, no se generaría una reproducción ampliada del producto de la economía de manera que provea el desarrollo de nuevas tecnologías y con ello incrementar la $83 v$ crecimiento y desarrollo con sustentabilidad ambiental: un enfoque de cuentas ecológicas productividad del capital y el trabajo.

\section{Sustentabilidad y desarrollo sustentable}

Antes de intentar definir el desarrollo sustentable es pertinente referirse al concepto de sustentabilidad y establecer algunas diferencias de este concepto con el primero. La sustentabilidad se relaciona con la sociedad y los sistemas ecológicos. Se puede identificar tanto con la actual generación como con las generaciones por venir. Ello deviene en un conjunto de preceptos vinculados a la gestión sustentable de los recursos naturales. 


\section{Análisis de los principales instrumentos económicos implementados en la política ambiental del Ecuador}

Vol. 3, núm. 1., (2019)

Nancy Roció Castillo Castro; Xavier Francisco Flores Torres; Víctor Hugo Navas Naranjo Elementos sobre la medición del desarrollo sustentable

Se establecen las limitaciones de la contabilidad nacional tradicional para medir el daño ambiental. El sistema de cuentas nacionales tradicional no se encuentra diseñado para registrar los costos asociados a la degradación y el agotamiento de los recursos naturales. Acerca de las limitaciones de la contabilidad nacional tradicional Claude (1997), desde la perspectiva del desarrollo sustentable, se refiere a las limitaciones de la contabilidad nacional y al debate que se desprende de las mismas por no haber tenido en cuenta una nueva dimensión que considere aspectos esenciales sobre el bienestar. Son varios los señalamientos críticos que se le hacen a la contabilidad nacional tradicional relacionados con sus limitaciones.

\section{Marco conceptual.}

\section{Sustentabilidad}

Se puede entender la sustentabilidad como un paradigma para pensar en un futuro en el que las consideraciones ambientales, sociales y económicas se balanceen en la búsqueda del desarrollo y una mejor calidad de vida (Colgrove, 2002). Esto quiere decir que con un buen equilibrio se puede tener un mejor porvenir y un mejor estilo de vida para los habitantes.

\section{Desarrollo.}

En este contexto, (London \& Formichella, 2006, págs. 17-32) expresó que para hablar del desarrollo de una sociedad hay que analizar la vida de quienes la integran, que no puede considerarse que hay éxito económico sin tener en cuenta la vida de los individuos que conforman la comunidad. Esto quiere decir que es un proceso donde las condiciones de bienes y 


\section{Análisis de los principales instrumentos económicos implementados en la política ambiental del Ecuador}

Vol. 3, núm. 1., (2019)

Nancy Roció Castillo Castro; Xavier Francisco Flores Torres; Víctor Hugo Navas Naranjo

servicios se encuentran en estado creciente y al alcance de todos los grupos de todos los grupos

que conforman la comunidad

\section{Desarrollo económico}

De acuerdo con Samuel Huntington el desarrollo político se define como la capacidad de un sistema político de hacer frente a crisis (políticas, sociales, económicas) que se presenten (1968). Es la manifestación político-social del proceso evolutivo general de la vida

\section{Economía}

Para Smith la Economía es "una de las ramas de la ciencia del legislador o del estadista" (Sharma, 2018). Smith delimita con más claridad el ámbito de esta rama del saber al describir dos objetos propuestos por la Economía, El primero, suministrar al pueblo un abundante ingreso o subsistencia, o, hablando con más propiedad, habilitar a sus individuos y ponerles en condiciones de lograr por sí mismos ambas cosas; el segundo, proveer al Estado o República de rentas suficientes para los servicios públicos. Procura realizar, pues, ambos fines, o sea enriquecer al soberano y al pueblo" (1776. La economía es el estudio de la producción, extracción, intercambio, distribución y consumo de todos los bienes y servicios que la sociedad necesita, así como la forma en que las sociedades se organizan, funcionan, prosperan y sobreviven. 


\section{Análisis de los principales instrumentos económicos implementados en la política ambiental del Ecuador}

Vol. 3, núm. 1., (2019)

Nancy Roció Castillo Castro; Xavier Francisco Flores Torres; Víctor Hugo Navas Naranjo

Marco referencial

Marco legal

Para esta investigación se revisaron artículos de la sección cuarta, Presupuesto General del Estado, la cual hace referencia al crecimiento del Ecuador y la sostenibilidad en el país.

Según el art. 280 del Plan Nacional de Desarrollo, indica: "El instrumento al que se sujetarán las políticas, programas y proyectos públicos; la programación y ejecución del presupuesto del Estado; y la inversión y la asignación de los recursos públicos; y coordinar las competencias exclusivas entre el Estado central y los gobiernos autónomos descentralizados. Su observancia será de carácter obligatorio para el sector público e indicativo para los demás sectores." (CNP, 2017)

Se basa en los logros de los "últimos 10 años" y pone en certidumbre la existencia de nuevos retos por alcanzar, en torno a tres ejes principales:

1) Derechos para todos durante toda la vida;

2) Economía al servicio de la sociedad;

3) Más sociedad, mejor Estado, que contienen a su vez tres objetivos nacionales de desarrollo que rompen con la lógica sectorial y dan cuenta de las prioridades que tiene el país.

Según el art. 293 indica la formulación y la ejecución del Presupuesto General del Estado la cual se sujetarán al Plan Nacional de Desarrollo. "Los presupuestos de los gobiernos autónomos descentralizados y los de otras entidades públicas se ajustarán a los planes regionales, 


\section{Análisis de los principales instrumentos económicos implementados en la política ambiental del Ecuador}

Vol. 3, núm. 1., (2019)

Nancy Roció Castillo Castro; Xavier Francisco Flores Torres; Víctor Hugo Navas Naranjo

provinciales, cantonales y parroquiales, respectivamente, en el marco del Plan Nacional de

Desarrollo, sin menoscabo de sus competencias y su autonomía.” (CNP, 2017).

Los gobiernos autónomos descentralizados se someterán a reglas fiscales y de endeudamiento interno, análogas a las del Presupuesto General del Estado, de acuerdo con la ley.

Art. 294 “La Función Ejecutiva elaborará cada año la proforma presupuestaria anual y la programación presupuestaria cuatrianual. La Asamblea Nacional controlará que la proforma anual y la programación cuatrianual se adecuen a la Constitución, a la ley y al Plan Nacional de Desarrollo y, en consecuencia, las aprobará u observará.

Art. 298.- Se establecen pre asignaciones presupuestarias destinadas a los gobiernos autónomos descentralizados, al sector salud, al sector educación, a la educación superior; y a la investigación, ciencia, tecnología e innovación en los términos previstos en la ley. Las transferencias correspondientes a pre asignaciones serán predecibles y automáticas. Se prohíbe crear otras pre asignaciones presupuestarias.

\section{Marco contextual}

Se realizará la investigación en Ecuador con la finalidad de analizar el desenvolvimiento, tanto económico, político y público, como la sustentabilidad del país. Ecuador, en los últimos años, se ha podía notar la gran variación del comportamiento de la economía ya que, a pesar de los auges económicos presentados en 1965, con el boom bananero y petrolero, el comportamiento del producto interno bruto, en el largo plazo, no refleja un crecimiento representativo. 


\section{Análisis de los principales instrumentos económicos implementados en la}

política ambiental del Ecuador

Vol. 3, núm. 1., (2019)

Nancy Roció Castillo Castro; Xavier Francisco Flores Torres; Víctor Hugo Navas Naranjo

La investigación describirá la organización, progreso y composición de los ingresos y gastos del sector público, así como proyecta las principales herramientas económicas de política ambiental implementadas en el Ecuador, para apoyar las tareas de financiamiento de acciones y el cumplimiento de objetivos de conservación de los recursos naturales.

Los datos analizados evidencian el grado de inflexibilidad existente en las finanzas públicas a nivel nacional, así como una fuerte dependencia respecto a las utilidades petroleras, lo que provoca una gran inestabilidad en los ingresos públicos. Se observa también que el margen de flexibilidad de la inversión ha sido desplazado hacia los gobiernos locales, quienes no han garantizado el cumplimiento de las tareas ambientales especificadas en sus mandatos institucionales.

El crecimiento económico contempla un largo camino que implica transformaciones en la estructura y dinámica económica, que generalmente constituyen el punto de partida en la generación de procesos de cambio estructural, en el caso del Ecuador la constante inestabilidad económica ha dificultado su ocurrencia y cuantificación. Identificar y analizar los factores económicos que participan o influyen en los procesos de cambio estructural e inciden en el crecimiento económico del Ecuador.

"El cambio estructural implica colocar en el centro de la dinámica de crecimiento los cambios cualitativos en la estructura productiva, tanto para una mejor inserción global como para un dinamismo interno virtuoso en el ámbito de la productividad y el empleo, para ello hay que procurar mayor participación de los sectores intensivos en conocimiento en la producción total. De esta forma, se promueve a lo ancho de la economía y el tejido social el desarrollo de 
Análisis de los principales instrumentos económicos implementados en la política ambiental del Ecuador

Vol. 3, núm. 1., (2019)

Nancy Roció Castillo Castro; Xavier Francisco Flores Torres; Víctor Hugo Navas Naranjo

capacidades, conocimientos y procesos de aprendizaje de manera coordinada con la producción y la inversión” (Yepez Hidalgo \& García Álvarez, 2016)

La identificación de cambios estructurales en la economía ecuatoriana es de difícil cuantificación debido a la presencia de un fuerte proceso de permanente inestabilidad económica, pero se puede afirmar que luego del primer cambio estructural de 1973 por la irrupción del petróleo no se han originado cambios estructurales propiamente dichos o entendidos como cambios en las estructuras productivas.

\section{Marco empírico}

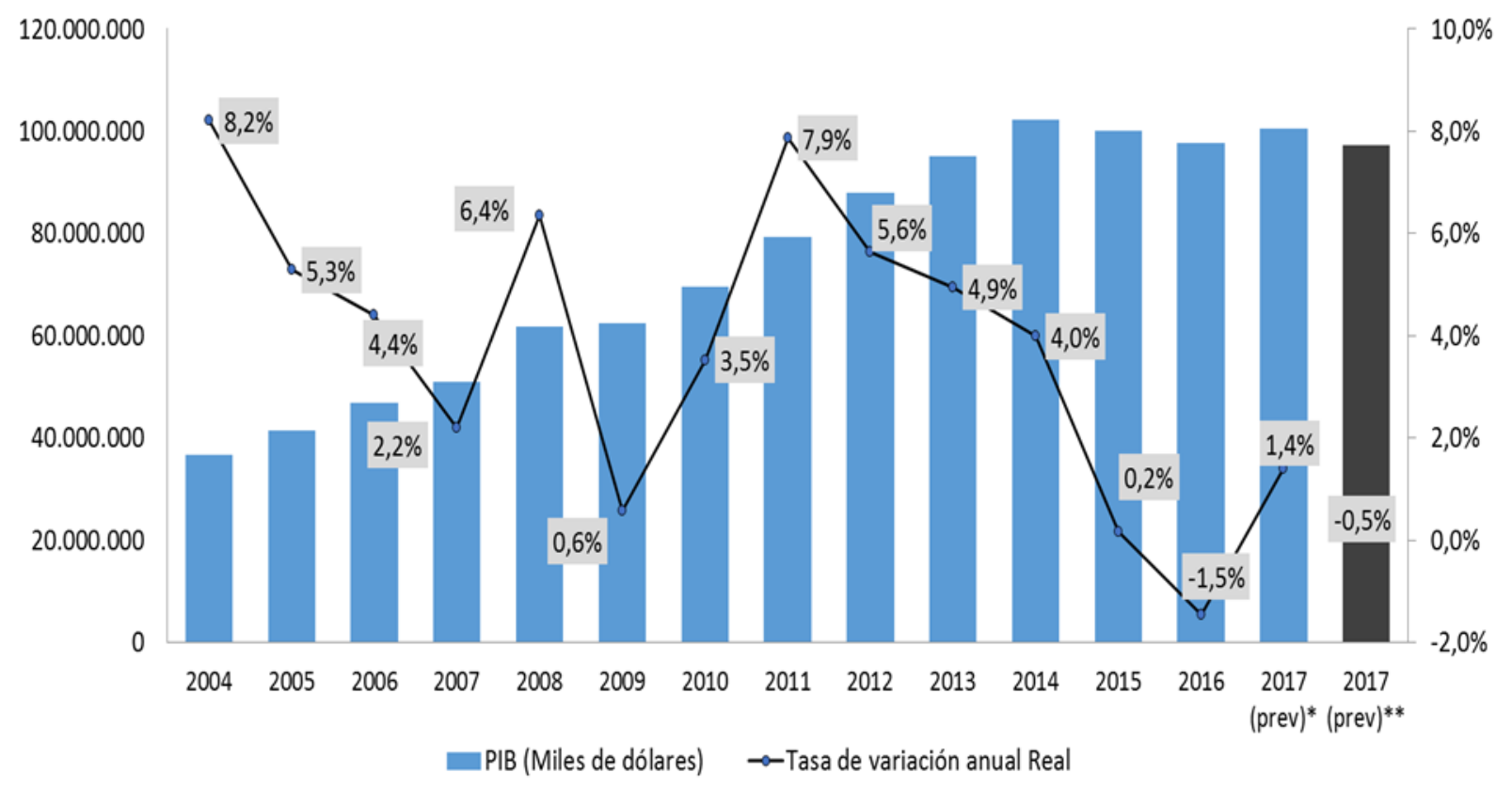

Ilustración 1 - Porcentajes de desarrollo del PIB 


\section{Análisis de los principales instrumentos económicos implementados en la política ambiental del Ecuador}

Vol. 3, núm. 1., (2019)

Nancy Roció Castillo Castro; Xavier Francisco Flores Torres; Víctor Hugo Navas Naranjo Porcentajes de desarrollo del PIB (miles de dólares)

Tasa de desarrollo

Como podemos observar en el grafico la economía del ecuador ha estado en constante desarrollo entre los años 2004 y 2014 en los cuales ha tenido un aumento porcentualmente bueno en comparación con los años anteriores. Aquí podemos observar que el aumento más alto del PIB es en año 2011 donde el aumento de este es del 7,9\% lo cual denota una visible economía en crecimiento. Pero a partir del año 2015 el crecimiento porcentualmente del PIB no solo se detiene, si no que baja en un $0,2 \%$. lo cual denota un declive en el desarrollo económico del ecuador a partir de ese año, ya que, desde el 2015 ocurrió un segundo declive en el PIB el cual fue del $1,5 \%$ disminuyendo no solo el capital del ecuador, si no, el poder der mercado del pueblo al aumentar constantemente la población y no mejorar el desarrollo económico de nuestro país.

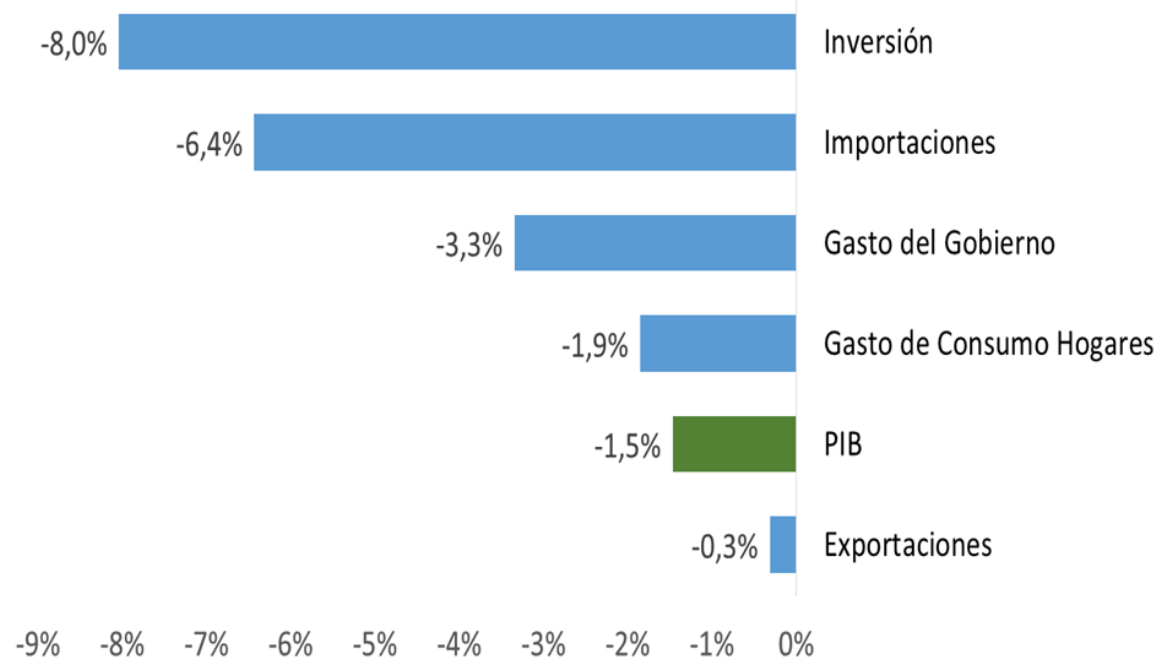

Ilustración 2 - Tasa del decrecimiento en el 2016 


\section{Análisis de los principales instrumentos económicos implementados en la política ambiental del Ecuador}

Vol. 3, núm. 1., (2019)

Nancy Roció Castillo Castro; Xavier Francisco Flores Torres; Víctor Hugo Navas Naranjo

Tasa de decrecimiento en el 2016

Como observamos en el cuadro anterior, en el 2016 el PIB tubo una disminución del 1,5\% la cual ha sido el declive más considerable en los últimos 14 años.

En este cuadro podemos observar cuales son los puntos en los que la economía del ecuador en el año 2016 tuvo inconvenientes y por consecuencia una disminución en el PIB. En la inversión y en las importaciones son los dos puntos donde hubo declive considerable siendo de un $8 \%$ y un $6,4 \%$ respectivamente. En el gasto del gobierno hubo una disminución del 3,3\% lo cual quiere decir que el gobierno no tuvo suficientes ingresos en este año y por ende tuvo que disminuir su presupuesto por la falta del recurso monetario. Las exportaciones cayeron en un 0,3\% lo cual a simple vista parece no tan malo, pero, al ser un país productor de materia prima, las exportaciones son un pilar fundamental en la economía del país, por lo cual un declive en las exportaciones significa una disminución en uno de los ingresos más importantes que tiene el estado ecuatoriano, el cual se ve reflejado en la disminución del gasto de consumo de hogares en un $1,9 \%$ debido a que las familias no tienen el presupuesto suficiente para poder satisfacer todas su necesidades y se ven obligadas a disminuir sus gastos para así tratar de sobrellevar el declive en la economía en la economía del país. 
Análisis de los principales instrumentos económicos implementados en la
política ambiental del Ecuador

Vol. 3, núm. 1., (2019)

Nancy Roció Castillo Castro; Xavier Francisco Flores Torres; Víctor Hugo Navas Naranjo

Crecimiento del PIB

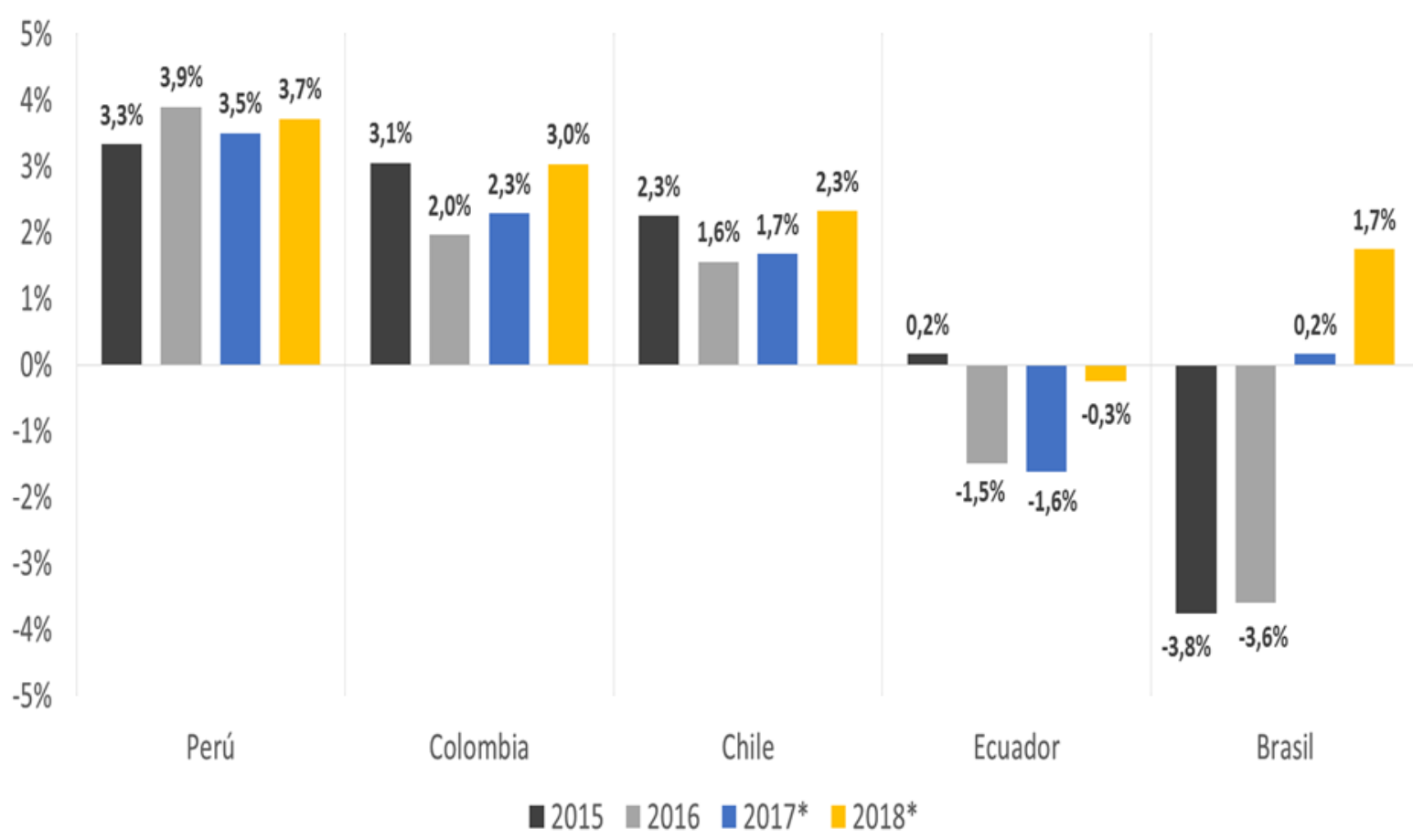

Ilustración 3 - Crecimiento del PIB

El desarrollo de la economía de nuestro país es uno de los más bajos en América latina, como podemos observar en éste cuadro, Ecuador solo muestra una disminución en el PIB en los años 2016, 2017 y 2018 en comparación con Perú, Colombia, Chile y Brasil los cuales tienen un aumento en sus PIB, lo que quiere decir que sus economías están en constante desarrollo y crecimiento.

Perú es uno de los países con un mejor desarrollo y crecimiento económico, en los últimos 4 años Perú tiene un promedio de crecimiento del PIB del 3,6\% de crecimiento por cada uno, le sigue Colombia con un promedio del 2,6\% de crecimiento del PIB en cada uno de los últimos cuatro años, de ahí viene Chile con un promedio del $2 \%$ por cada año. 
Análisis de los principales instrumentos económicos implementados en la política ambiental del Ecuador

Vol. 3, núm. 1., (2019)

Nancy Roció Castillo Castro; Xavier Francisco Flores Torres; Víctor Hugo Navas Naranjo

Por ende, se nota que ecuador no tiene un buen desenvolvimiento económico ya que se refleja en el poder de mercado de sus habitantes que su economía no solo se ha estancado, sino que está en declive afectando no solo al gobierno, sino a las empresas y al estado en general, disminuyendo el trabajo, aumentando los precios y decayendo el producto interno bruto

\section{Inflación}

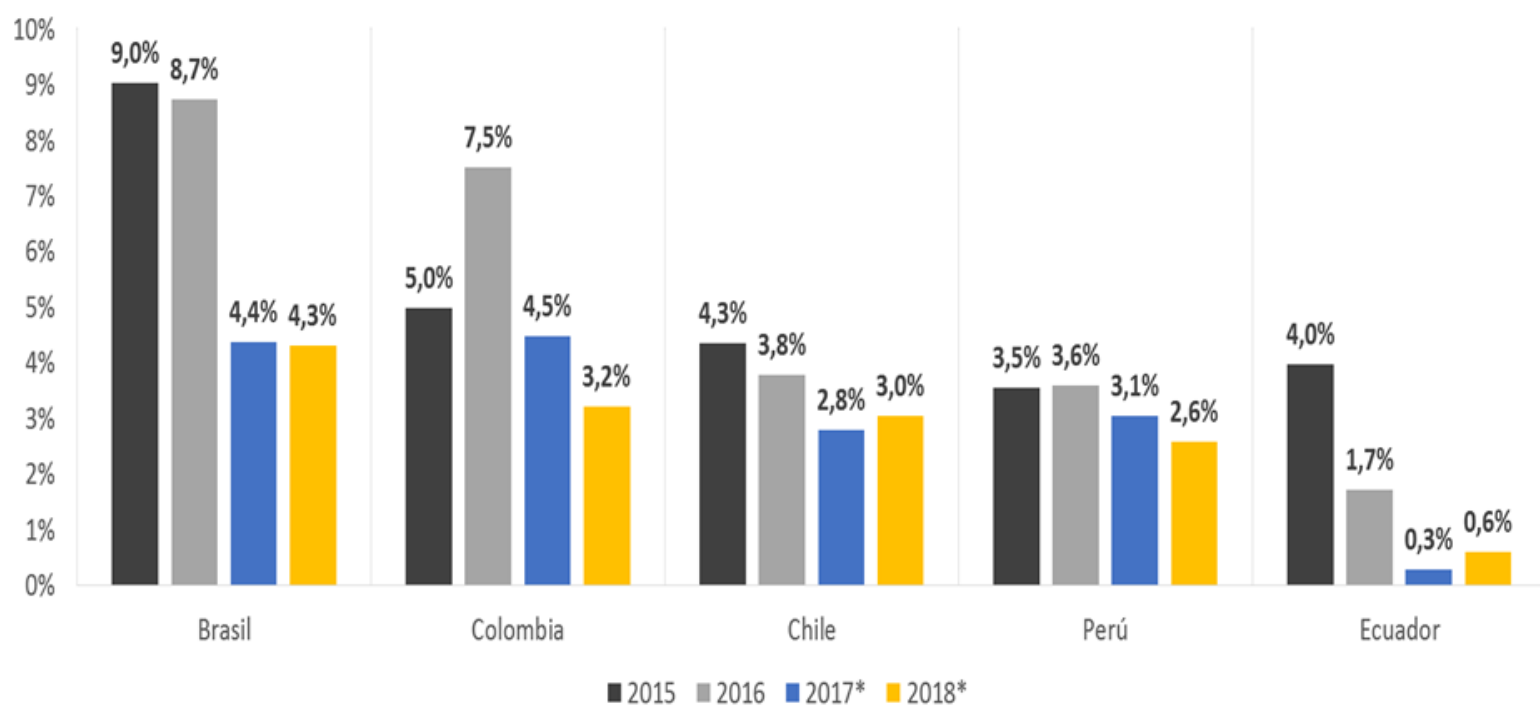

\section{Ilustración 4 - Inflación}

Sin embargo, como podemos observar en el cuadro, Ecuador es uno de los países con el porcentaje de inflación más bajo de Latinoamérica. Esto se debe a que en ecuador no se maneja una moneda nacional, la moneda oficial es el dólar el cual esta administrado por los Estados Unidos De América y son de quienes depende el valor de la moneda.

Ésta situación es la que permite que ecuador tenga una inflación porcentualmente muy baja en comparación con los demás países que se encuentran en el gráfico, y es la razón por la 


\section{Análisis de los principales instrumentos económicos implementados en la política ambiental del Ecuador}

Vol. 3, núm. 1., (2019)

Nancy Roció Castillo Castro; Xavier Francisco Flores Torres; Víctor Hugo Navas Naranjo

cual la economía ecuatoriana se sostiene y no ha decaído como otros países con un desarrollo económico casi igual al ecuatoriano.

\section{Conclusiones.}

Para finalizar tenemos que Ecuador fue reconocido como modelo de desarrollo sostenible para la erradicar la pobreza y reducir la desigualdad, en el marco de la XI Reunión Anual de las Naciones Unidas, Organizaciones Sociales y Sociedad Civil. La promoción del sistema de economía social y solidaria y el reconocimiento de los derechos de la naturaleza son importantes pasos hacia una Economía Ecológica, concepto por el cual trabajamos en Ecuador.

El crecimiento económico contempla un largo camino que implica transformaciones en la estructura y dinámica económica, que generalmente constituyen el punto de partida en la generación de procesos de cambio estructural, la producción y consumo sustentable se han convertido en un mecanismo para consolidar el cambio de la matriz productiva, pues esto suman a la implementación de tecnologías limpias, destacando las buenas prácticas ambientales, con el propósito de reducir la contaminación y optimizar de esta manera el uso de nuestros recursos naturales, en todas sus fases o ciclo de vida de producto, reduciendo el impacto negativo al ser humano y su entorno natural.

\section{Bibliografía.}

CNP. (2017). Plan Nacional del Buen Vivir. Quito: Consejo Nacional de Planificación.

Colgrove, J. (2002). The McKeown Thesis: A Historical Controversy and Its Enduring Influence. Am J Public Health, 92(5), 725-729. 


\section{Análisis de los principales instrumentos económicos implementados en la política ambiental del Ecuador}

Vol. 3, núm. 1., (2019)

Nancy Roció Castillo Castro; Xavier Francisco Flores Torres; Víctor Hugo Navas Naranjo

London, S., \& Formichella, M. (2006). El concepto de desarrollo de Sen y su vinculación con la Educación. Economía y Sociedad, 9(17), 17-32.

Sharma, R. (29 de 12 de 2018). Adam Smith: The Father of Economics. Obtenido de Investopedia: https://www.investopedia.com/updates/adam-smith-economics/

Yepez Hidalgo, R., \& García Álvarez, S. (2016). Analizando la senda y los factores de crecimiento del Ecuador desde 1965 hasta el año 2013. Carrera de Economía. Quito: Universidad Central del Ecuador. 